\title{
Variation of Body Mass Index and Peak Expiratory Flow Rate among Medical Students of CMH Lahore Medical College
}

\section{Farhat Ijaz, ${ }^{1}$ Imtiaz Bashir, ${ }^{2}$ Azal Ikhlaq, ${ }^{3}$ Farida Hafeez, ${ }^{4}$ Rana Khurram Aftab, ${ }^{5}$ Sana Asif Malik ${ }^{6}$}

\begin{abstract}
Objective: To find out the variation of body mass index and peak expiratory flow rate among medical students of Combined Military Hospital, Lahore Medical College, Lahore, Pakistan.

Methods: A Cross-sectional study was conducted by undergraduate students of CMH, at CMH Lahore Medical College and Institute of Dentistry in September 2019 after Ethical approval from the Ethical Review Committee of the same institution. There were 1381 st year medical students. Demographic profiles of all the students were taken and height, weight, BMI, PEFR were measured. Participants were classified on the basis of their BMI values. Underweight (BMI $<18.5)$, normal weight $(18.5 \leq \mathrm{BMI} \leq 24.9)$, overweight $(25 \leq \mathrm{BM} \leq$ $29.9)$, and obese (BMI $\geq 30)$. Correlational tests were applied to find out any statistically significant correlations. Ap value less than 0.05 was considered significant.

Results: The mean BMI in females was 23.16 \pm 6.01 corresponding with that of mean PEFR value $325.23 \pm 62.30$ whereas in males the mean BMI was 22.65 \pm 3.11 corresponding with that of mean PEFR value 433.97 \pm 101.84 . There is a statistically significant variation in PEFR with gender $(r=0.540, p=0.001)$ which can be explained on ethnic backgrounds. Males had a higher PEFR than females. However, there was no significant correlation between BMI and PEFR. Also, gender was not related to BMI.

Conclusion: In our study, PEFR is not affected by variation in BMI. However, gender is associated with PEFR. Males have a higher PEFR than females. This can be explained on the basis of ethnicity. BMI is not associated with gender. A large sample size with more accurate calculation of PEFR is needed for better evaluation.

Keywords: Obesity and lung function, PEFR and BMI, BMI and gender

How to cite: Ijaz F, Hafeez F, Bashir I, Aftab RK, Malik SA.Variation of body mass index and peak expiratory flow rate among medical students of CMH Lahore medical college. J SIMS Esculapio. 2021;17(1):100-103

DOI: https://doi.org/10.51273/esc21.2517120
\end{abstract}

\section{Introduction}

B ody Mass Index (BMI)- which is the measurement of weight relative to height- is the tool used worldwide to classify an individual as Overweight, Underweight or Obese. People having a BMI greater

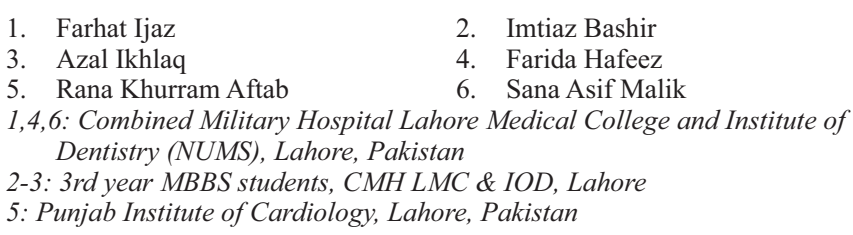

Dr. Farhat Ijaz, Department of Physiology ,Combined Military Hospital Lahore Medical College \& Institute of Dentistry, Lahore, Pakistan. Email :farhat_khurram_rana@cmhlahore.edu.pk
Submission Date:

1st Revision Date:

Acceptance Date:
$01-12-2020$

$09-12-2020$

20-01-2021 than $25 \mathrm{~kg} / \mathrm{m}^{2}$ are considered "Overweight" while those having greater than $30 \mathrm{~kg} / \mathrm{m}^{2}$ are considered as "Obese". Still, BMI is not a gold standard for the measure of degree of fatness in an individual. ${ }^{1}$ An Emerging Global Health Peril is Obesity which has equally affected both developing and developed countries. Pakistan stands 165th /194 in the ranking of "World Fattest Countries" with 22.2\% people crossing the subliminal level of Obesity. ${ }^{2}$ Previous studies have shown a large number of medical students to be Overweight/Obese. Factors responsible for this include sedentary lifestyle, prolonged sitting, genetic influence, over-consumption of fast food, over-eating due to exam stress, and least physical activity.,

Obesity- often referred to as mother of all diseasesputs a person to the peril of severe ailments including 
espiratory diseases. ${ }^{5}$ Increased fat levels decrease the lung compliance leading to various problems such as breathlessness and decreased exercise tolerance. ${ }^{6}$

Peak Expiratory Flow Rate (PEFR) is defined as the maximal flow of air that can be attained after maximum inhalation during a forceful exhalation. It is used as a tool to test the flow of air through the airway of a patient and to check for any obstruction in the lungs. The value of PEFR may vary with gender, age, race, and environment. ${ }^{7}$ Various studies have demarcated the Forced Expiratory Volume taken at the 1st second of forced exhalation (FEV1) to be a better indicator for airway obstruction than PEFR. Still, PEFR is preferable due to its portability, easy handling, patient feasibility and cost effectiveness. The Wright's Peak Flow Meter is commonly used in laboratories for PEFR measurement. ${ }^{8}$

This descriptive study aimed at assessing and documenting the BMI and PEFR of the newly enrolled medical undergraduates to describe the general health status of medical students. Previous studies ${ }^{7,9,10,13}$ indicate both positive and negative association of BMI with PEFR. We also aimed to re-check this correlation in our sample. Ethnic backgrounds and social factors are not considered in this study.

\section{Methods}

A cross sectional study was conducted by undergraduate students of $\mathrm{CMH}$, at $\mathrm{CMH}$ Lahore Medical College and Institute of Dentistry in Sept. Ethical Approval was taken from Ethical Review Committee of CMH Lahore Medical College prior to the conduction of study. All the participants were assured that their identity will not be disclosed, and the data collected will be used only for research purposes. They were told that they could withdraw from the study any time if they have concerns. All their queries were addressed I details.

All students of 1st Year MBBS were included, the participants were between the ages of 17 and 22. The study variable were height, weight, BMI and PEFR. Protocols of the procedure were described clearly to all the students prior to the conduction experiment.

Vitals (blood pressure, pulse, respiratory rates) were measured for all the students prior to the measurement of PEFR. All the measurements were done with care under observation of the research team. Heights were measured in centimeters using stadiometer, and weights were measured using standard calibrated weighing scales. PEFR was measured with Wright's Peak Flow Meter in Liter/minutes. Experiments were repeated thrice, and a mean was taken to reduce any error.

Demographic profiles of all the students, height, weight, BMI, and PEFR were recorded in the form of descriptive data. Pearson's correlation was applied to find out any significant correlations. P-value $\leq 0.05$ was taken significant. Statistical Package for Social Sciences (SPSS v 25) was used for statistical analysis.

\section{Results}

Table 1 shows the distribution of study population. Male participants were larger in percentage and had a mean age of $18.93+1.045$. Females had a mean age of $18.88+0.992$.

Table 2. shows the variation of BMI with PEFR of the subjects. Table 3 and Table 4 provide a correlation of these values with gender. Only three out of 138 were

obese while 15 were underweight and 35 were overweight. A statistically significant correlation was found between PEFT and BMI in the underweight and normal-weight grouped people $(\mathrm{r}=0.573, \mathrm{p}=0.02$ in underweight people, and $\mathrm{r}=0.240, \mathrm{p}=0.02$ in normal-weight people). However, no significant correlation between BMI and PEFR was found in overweight and obese people.

The statistical analysis using Pearson's Correlation shows that there is no correlation between BMI and PEFR and also that gender is not related to BMI.

Table 2: Variation of PEFR with BMI

\begin{tabular}{lccc}
\hline \multicolumn{4}{l}{ Relationship between PEFR and BMI } \\
\hline BMI Range & Mean PEFR & N & Std. Deviation \\
Less than 18.5 & 389.33 & 15 & 77.318 \\
$18.5-24.9$ & 380.00 & 85 & 103.003 \\
$25-29.9$ & 388.00 & 35 & 110.155 \\
30.0 and Above & 366.67 & 3 & 28.868 \\
Total & 382.75 & 138 & 100.805 \\
\hline
\end{tabular}

However, gender has a statistically significant correlation with PEFR. 


\section{Discussion}

Obesity is an emerging problem in Pakistan, not least in women and young adults. This is due to sedentary

Table 3: Variation of BMI and PEFR with Gender

\begin{tabular}{ccc}
\hline Gender, BMI and PEFR & \\
\hline Gender & Mean BMI & Mean PEFR* \\
Male & $22.65 \pm 3.110$ & $433.97 \pm 101.84$ \\
Female & $23.16 \pm 6.013$ & $325.23 \pm 62.302$ \\
\hline
\end{tabular}

*Variation of PEFR with Gender $(\mathrm{r}=0.540, \mathrm{p}=0.001)$

lifestyles and unhealthy food habits. Our study demonstrates that PEFR in males was greater than in females. One explanation for this finding is based on height. A statistically significant relationship $(\mathrm{p}<0.01)$ is found between height and PEFR when studied for the combined data of both genders. In our study population, males were generally taller than females (the mean height of males was $173.28+6.67 \mathrm{~cm}$ and of the females was $158.62+7.93 \mathrm{~cm}$ ). Given that (1) height is positively related to PEFR and (2) males are taller in this study population, this might account for the higher PEFR findings in males than females. Similar findings have been reported previously. ${ }^{16}$ An alternate explanation suggests that decreased PEFR in females might also be associated with decreased physical activity and ethnic background, but this is limited in its explanatory powers and was not tested in our work and thus cannot be objectively remarked upon without further study.

The results of our study are consistent with some of the studies done earlier and are in contrast with others. Our results show that there is no correlation between BMI and PEFR. This is in consistence with the study done by Harpreet ${ }^{7}$ and Saraswathi Ilango ${ }^{10}$ and in contrast with Sudha ${ }^{9}$ and Dharamshi. ${ }^{13}$ This contrast can be explained by the fact that the studies showing contrast results had a skewed sample i.e., increased population of either male or female gender.

Some studies ${ }^{10}$ hypothesize that body fat distribution might have effect on Lung functioning. Abdominal fat content might interfere with the mobilization of the diaphragm and thus decreasing lung functionality. They also hypothesize that lung function might be different in females and males because of different body fat distributions i.e., males have android obesity and females have gynecoid obesity. However, no significant experimental studies support this notion. Our study is also in contrast with this hypothesis. It indicates no correlation between BMI and PEFR.
In contrast to other studies, ${ }^{11,12}$ the BMI values were not found to be related with gender. Both males and females had almost an equal mean of BMI. The only significant correlation was that PEFR was higher in males than the females similar to other studies. ${ }^{12,13}$ This can be explained on the basis of ethnic and cultural differences. Ethnicity is considered as an important factor that affects PEFR. ${ }^{7}$ Most of the male participants of our study had a strenuous physical activity as a part of their daily routine. This might be a reason of marked differences in the PEFR values of males and females.

\section{Limitations}

A larger sample size with a detailed insight of ethnic background is needed to get more accurate results. Moreover, Wright's Peak Flow Meter is less accurate than its counterparts. This might put an error in the calculation of results. A more accurate Peak Flow Meter can be used to level up the accuracy of the acquired data. ${ }^{15}$

\section{Conclusion}

The study concludes that PEFR is not affected by variation in BMI. However, gender is associated with PEFR. Males have a higher PEFR than females. This can be explained on the basis of ethnicity. BMI is not associated with gender. A large sample size with more accurate calculation of PEFR is needed for better evaluation.

\section{Conflict of Interest: $\quad$ None}

\section{References}

1. World Health Organization. World Health Organization fact sheet: obesity and overweight. [cited 2020 October 24]. Available from: https:// www.who.int/ news-room/fact-sheets/detail/obesity-and-overweight.

2. Streib L. World's fattest countries. Forbes [https:// www. forbes.com/2007/02/07/worlds-fattestcountries-forbeslife-cx_1s_0208worldfat.html?sh= 2530060464f1].2021 Feb.

3. Bakr EM, Ismail NA, Mahaba HM. Impact of life style on the nutritional status of medical students at Ain Shams University. The Journal of the Egyptian Public Health Association. 2002 Jan 1;77(1-2):29-49.

4. Ganasegeran K, Al-Dubai SA, Qureshi AM, Al-Abed AA, Rizal AM, Aljunid SM. Social and psychological factors affecting eating habits among university students in a Malaysian medical school: a cross-sec- 
tional study. Nutrition journal. 2012 Dec;11(1):1-7.

6. Salome CM, King GG, Berend N. Physiology of obesity and effects on lung function. Journal of applied physiology. 2010 Jan;108(1):206-11.

7. Kaur H, Singh J, Makkar M, Singh K, Garg R. Variations in the peak expiratory flow rate with various factors in a population of healthy women of the malwa region of Punjab, India. Journal of clinical and diagnostic research: JCDR. 2013 Jun;7(6):1000.

8. Mittal S, Gupta S, Kumar A, Singh KD. Regression equations for peak expiratory flow in healthy children aged 7 to 14 years from Punjab, India. Lung India: Official Organ of Indian Chest Society. 2013 Jul; 30(3): 183.

9. Sudha D, Chandra Selvi E, Saikumar P. Correlation of nutritional status and peak expiratory flow rate in normal South Indian children aged 6 to 10 years. IOSR Journal of Dental and Medical Sciences (JDMS). 2012;2:11-6.

10. Saraswathi I, Christy A, Saravanan A, Dr Prema S. Correlation of obesity indices with peak expiratory flow rate in males and females. IOSR Journal of Pharmacy. 2014;4(2):21-7.

11. Chen J, Yi H, Liu Z, Fan Y, Bian J, Guo W, et al., Factors associated with being overweight among Inner Mongolia medical students in China. BMJ open. 2013 Dec 1;3(12).

12. Arthur JP, Macstephen AO. Correlation between Body Mass Index and Peak Expiratory Flow Rate of an Indigenous Nigerian Population in the Niger Delta Region. Research Journal of Recent Sciences ISSN.; 2277:2502.

13. Dharamshi HA, Faraz A, Ashraf E, Ali AA, Rameez MA, Abidi SM, Ullah S, Rehman MU, Nafees T. Variation of Peak Expiratory Flow Rate with Body Mass Index in Medical Students of Karachi, Pakistan. International Archives of Medicine. 2015 Jun 2;8.

15. Miller MR, Dickinson SA, Hitchings DJ. The accuracy of portable peak flow meters. Thorax. $1992 \mathrm{Nov}$ 1;47(11):904-9.

16. Rochester DF, Enson Y: Current concepts in the pathogenesis of the obesity-hypoventilation syndrome. Mechanical and circulatory factors. Am J Med. 1974, 57:402-420. 10.1016/0002-9343(74)90135-1
Authors Contribution
IF,IA,AKR: Conceptionlization of Project
BI,IA : Data Collection
AKR,MAS: Literature Search
HF,AKR: Statistical Analysis
IF,HF: Drafting, Revision
IF,IA: Writing of Manuscript 